\title{
Response of "Grandnain"Banana to Humic Acid, Potassium and Magnesium Fertilization
}

\author{
Mohamed Ragab El-Shenawi, Hoda S.H. Aly and Badran, A. F. Mohamed ${ }^{1}$
}

\begin{abstract}
This study was carried out in a private orchard, at Kafr El-Dwar, El-Behera Governorate, on "GrandNain" banana plants (Musa cavendishii Lamb.) during the 2006/2007 and 2007/2008 growing seasons. Twelve treatments of different Humic acid (HA), potassium (K) and Magneisum (Mg) HA:K:Mg ratios were applied. The results indicated that there were great effects on growth aspects, yield, physical and chemical properties and leaf mineral content with varying HA:K:Mg ratios. Since increasing HA:K:Mg rates from 0 to $10 \mathrm{gm} / \mathrm{plant}$, $\mathrm{K}$ from 0 to $300 \mathrm{gm} /$ plant and $\mathrm{Mg}$ from 0 to $150 \mathrm{gm} / \mathrm{plant} / \mathrm{year}$ caused a remarkable promotion on all studied parameters. Leaf potassium and magnesium contents were increased with increasing the applied potassium and magnesium fertilizer rates. On the other hand, results did not show a constant trend due to different treatments in respect with phosphorus, but did not affect nitrogen content. The best treatment was obtained with using (HA:K:Mg) at rate of $10 \mathrm{gm} /$ plant Humic acid, $300 \mathrm{gm}$ potassium sulfate and 150 gm magnesium sulfate for all studied parameters.
\end{abstract}

\section{INTRODUCTION}

Banana (Musa cavendishi Lamb.) is considered as one of the most important fruit in tropical zones of the world. Banana fruits are highly appreciated by Egyptian consumers and are considered good source of energy and vitamins A, B6 and C. Gross return of banana is very high and environmental conditions in Egypt are suitable for growing and production (El-Shenawi, 2000).

It is well known that banana needs large amounts of fertilizers especially nitrogen and potassium. Moreover, it draws nutrients from a very limited soil depth because of its shallow root system (Saleh, 1996). On the other hand, the major problem facing banana growers is the high costs of excessive manufactured fertilizers needed for banana plants. Besides, these chemical fertilizers cause air, soil and water pollution during their production and utilization. The pollution of the soil and water resulted from leached chemical fertilizers into the soil, which transfer through the plants to the human and causes serious diseases (El-Shenawi and El-Sayed, 2005). For banana plants, using banana compost and chicken manure induced similar results with the recommended dose of mineral nitrogen fertilizer and gave the best fruit characteristics, (Abd El-Naby and Gomaa, 2000 and Gomaa and Abd El-Naby, 2000). On the other hand, many commercial products containing humic acid (HA) have been promoted for use on various crops, Liu et al. (1998). Benefits ascribed to the use of humic acid, particularly in low organic matter, alkaline soil, include increasing nutrient uptake, tolerance to drought and temperature extremes, stimulating activity of beneficial soil micro organisms and availability of soil nutrients (Seen and Kingman, 1973; and Russo and Berlyn, 1990). Humic materials may also increase root growth in a manner similar to auxins, (Donnell, 1973 and Tatini et al., 1991). Liquid fertilizer containing humic acid increased apple fruit weight, yield and soluble solids content (Li et al., 1999). Saad and Atawia (1999) studied the effect of potassium fertilizers treatments on the growth, yield and fruit quality of GrandNain banana, they found that the treatment of $800 \mathrm{gm} \mathrm{K}_{2} \mathrm{O}$ /plant/year seems not only to be the promising treatment to produce the highest vegetative growth and yield but also to improve the fruit quality. Moreover, potassium in required in great amounts as reported by different researchers. Lahave (1976) found that increasing the amount of manure caused early flowering and reduced maturation period. Application of both $\mathrm{KNO}_{3}$ and organic manure were necessary to obtain optimal yield and early flowering of Williams banana grown from suckers (Lahave et al., 1981). All plantations given potassium produced better yields than the control.

Humic substances are ubiquitous in the environment. Their importance in agriculture and soil sciences has been acknowledged for over 150 years. Purchase 1997, Humic acid contains Sulfur, Nitrogen and Phosphorus in varying amounts. It also contains metals such as $\mathrm{Ca}, \mathrm{Mg}, \mathrm{Cu}, \mathrm{Zn}$ etc. A substantial fraction of the mass of the humic acids is in carboxylic acid functional groups, which endow these molecules with the ability to chelate positively charged multivalent ions (Mg++, $\mathrm{Ca}++, \mathrm{Fe}++$ and most other "trace elements" of value to plants, as well as other ions that have no positive biological role, such as $\mathrm{Cd}++$ and $\mathrm{Pb}++$.) This chelation of ions is probably the most important role of humic acids with respect to living systems.

The aim of this study is to study the effect of humic acid, potassium and magnesium fertilization on vegetative growth, yield, fruit quality and some leaf mineral contents of GrandNain banana in order to reduce

\footnotetext{
${ }^{1}$ Hort. Res. Institute, Agric. Center, Egypt.

E-mail: hoda_saad23@yahoo.com

Received December21, 2008, Accepted December 28, 2008
} 
the costs of fertilizers increase the yield and improve the fruit quality.

\section{MATERIALS AND METHODS}

The present study was carried out during two successive seasons of 2006/2007 and 2007/2008 in a private orchard at Kafr El-Dwar, El-Behera Governorate on GrandNain banana plants (Musa Cavendishii Lamb.). Plants were grown at $3 \times 3 \mathrm{~m}$., apart in clay soil. The suckers were planted in March 2005 (Two offshoots/mother plant) 900 plants/fed. The experiment started on the first ratoon and its suckers were chosen on the $1^{\text {st }}$ week of July 2005 as well as on the second ratoon and its suckers were chosen at the same time in 2006. The stools were thinned and two suckers were left for fruiting in the following season. In addition, two plants were left to give the crop in the current season. In both seasons, organic manure was added in December at the form of chicken manure with rate of $15 \mathrm{~m}^{3} / \mathrm{fed}$. Nitrogen fertilizers was added to the plants on twelve equal split doses every 15 days intervals starting on April $1^{\text {st }}$ until October in form of ammonium sulfate $(20.5 \% \mathrm{~N})$. Calcium super mono phosphate $\left(16 \% \mathrm{P}_{2} \mathrm{O}_{5}\right)$ was also added every season per feddan once in December with rate of ton/fed. Banana plants were irrigated by surface irrigation system every 8-10 days through the growing season. Each treatment was represented by 9 holes distributed in Complete Randmized Block Design; three of each block received one of the following treatments:

\begin{tabular}{cccc}
\hline $\begin{array}{c}\text { Treatment } \\
\text { No. }\end{array}$ & $\begin{array}{c}\text { Humic } \\
(\mathbf{g m})\end{array}$ & $\begin{array}{c}\text { Potassium } \\
\text { sulfate }(\mathbf{g m})\end{array}$ & $\begin{array}{c}\text { Magnesium } \\
\text { sulfate }(\mathbf{g m})\end{array}$ \\
\hline 1 (Control) & 0 & 0 & 0 \\
2 & 0 & 100 & 50 \\
3 & 0 & 200 & 100 \\
4 & 0 & 300 & 150 \\
5 & 5 & 0 & 0 \\
6 & 5 & 100 & 50 \\
7 & 5 & 200 & 100 \\
8 & 5 & 300 & 150 \\
9 & 10 & 0 & 0 \\
10 & 10 & 100 & 50 \\
11 & 10 & 200 & 100 \\
12 & 10 & 300 & 150 \\
\hline
\end{tabular}

Soil fertilizer (humer $86 \%$ potassium hummate), the humer was added at one dose at the first week of May. Potassium sulfate $\left(48 \% \mathrm{~K}_{2} \mathrm{O}\right)$ was added at three equal doses (April, June and August). Magnesium sulfate $\left(48 \% \mathrm{Mg}_{2} \mathrm{O}\right)$ was added in one dose at first week May, in both seasons.

Standard annual measurements carried of banana plants vigor; yield and leaf nutrients contents were made. The following parameters were used to evaluate tested treatments:

\section{Vegetative growth:}

At the bunch shooting stage, pseudostem length $\mathrm{cm}$ (PSL), pseudostem girth, cm (PG), number of green leaves (NGL), number of days from shooting to harvest (NDSH) were counted and total leaf area (TLA) was calculated according to Ahmed and Morsy (1999), using the formula

TLA $=0.67(1 \times \mathrm{w})+107.15$

Where: $\mathrm{T}=$ Total $\mathrm{L}=$ leaf $\quad \mathrm{A}=$ area

l = length $\quad \mathrm{W}=$ width

\section{Yield, bunch parameters:}

At harvesting time, bunch weight in $\mathrm{Kg}$ (BW) and harvested yield (BHY), tone/fed., were estimated. On the other hand, number of hands per bunch (NHB), number of fingers per bunch (NFB) were computed as bunch characteristics.

\section{Physical and chemical fruit quality:}

Average finger diameter $(\mathrm{cm})$ was measured as physical fruit quality. Total acidity $\%$ (expressed $\%$ as gm malic acid/100 gm pulp), total soluble solids \%, starch \% and total sugars \% were determined according to A.O.A.C. 1995 as indicators of chemical fruit quality.

\section{Leaves nutrient contents:}

Leaf samples were collected at bunch shooting stage from the third upper leaf in the succession of leaves from the top of the plant as recommended by (Hewit, 1955 and Lahave and Turner, 1989). Macro elements (N, P, K and $\mathrm{Mg}$ ) were determined in banana leaves. Leaf samples were washed, then dried at $70^{\circ} \mathrm{C}$ ground and wet digested using $\mathrm{H}_{2} \mathrm{SO}_{4}$ and $\mathrm{H}_{2} \mathrm{O}_{2}$ (FAO, 1980). N, P, K and $\mathrm{Mg}$ concentrations were determined using semiautomatic nitrogen distillation unit, spectrophotometer 21D and Jenway flame photometer, respectively, (Westerman, 1990). Magnesium was determined against a standard using Perkin Elmer 305 B Atomic Absorption Spectrophotometer.

Soil samples were taken at $0-30 \mathrm{~cm}, 30-60 \mathrm{~cm}$ and $60-90 \mathrm{~cm}$ from soil surface orchard for routine analysis and the data is listed in Table (1).

The obtained data during both studied growing seasons were statistically analyzed according to Sendecor and Cochran (1990) and LSD test at 0.05 levels was used.

\section{RESULTS AND DISCUSSION}

\section{Effect of Humic acid, $\mathrm{K}$ and $\mathrm{Mg}$ fertilization on growth:}

The data in Table (2) indicated that all treatments markedly increased pseudestem length, pseudestem girth, number of green leaves/plant and leaf area as compared with control. Moreover, treatment $\mathrm{HA}: \mathrm{K}: \mathrm{Mg}$ 
Table 1. Mechanical and chemical soil analysis

\begin{tabular}{|c|c|c|c|}
\hline Characteristics & $0-30$ & $30-60$ & $60-90$ \\
\hline \multicolumn{4}{|c|}{ Mechanical analysis } \\
\hline Sand $\%$ & 20.25 & 16.42 & 21 \\
\hline Silt \% & 34.80 & 31.38 & 34 \\
\hline Clay \% & 44.95 & 52.20 & 45 \\
\hline Soil texture & Clay & Clay & Clay \\
\hline \multicolumn{4}{|c|}{ Chemical analysis } \\
\hline O.M. \% & 2.2 & 2.04 & 1.80 \\
\hline $\mathrm{pH}$ & 7.7 & 7.90 & 8.23 \\
\hline E.C. $\mathrm{mmhos} / \mathrm{cm}(1: 5)$ & 0.55 & 0.57 & 0.58 \\
\hline \multicolumn{4}{|c|}{ Cations and anions meq/L } \\
\hline $\mathrm{Ca}^{++}$ & 2.60 & 2.80 & 2.70 \\
\hline $\mathrm{Mg}^{++}$ & 2.20 & 2.10 & 2.00 \\
\hline $\mathrm{Na}^{+}$ & 0.70 & 0.78 & 0.77 \\
\hline $\mathrm{K}^{+}$ & 0.05 & 0.04 & 0.03 \\
\hline $\mathrm{HCo}_{3}^{-}$ & 5.00 & 5.0 & 4.9 \\
\hline $\mathrm{Cl}^{-}$ & 0.35 & 0.54 & 0.53 \\
\hline $\mathrm{So}_{4}^{--}$ & 0.22 & 0.18 & 0.17 \\
\hline
\end{tabular}

Table 2. Effect of Humic acid, $\mathrm{K}$ and $\mathrm{Mg}$ on some growth aspects of GrandNain banana 2006/2007 and 2007/2008 seasons

\begin{tabular}{|c|c|c|c|c|c|}
\hline $\begin{array}{l}\text { Treatments } \\
\text { (HA:K:Mg) } \\
\text { gm/plant/year }\end{array}$ & $\begin{array}{l}\text { Pseudstem } \\
\text { Length }(\mathbf{c m})\end{array}$ & $\begin{array}{l}\text { Pseudstem } \\
\text { girth }(\mathbf{c m})\end{array}$ & $\begin{array}{l}\text { Number of green } \\
\text { leaves/plant }\end{array}$ & $\begin{array}{ll}\begin{array}{l}\text { Leaf } \\
\left(\mathbf{m}^{2}\right)\end{array} & \text { area }\end{array}$ & $\begin{array}{l}\text { Number of days } \\
\text { from shooting to } \\
\text { harvest }\end{array}$ \\
\hline \multicolumn{6}{|c|}{$2006 / 2007$} \\
\hline $0: 0: 0$ & $243.0 \mathrm{~g}$ & $65.3 \mathrm{f}$ & $10.0 \mathrm{f}$ & $22.0 \mathrm{~g}$ & $195.3 \mathrm{a}$ \\
\hline 0:100:50 & $257.0 \mathrm{e}$ & $71.0 \mathrm{e}$ & $11.7 \mathrm{e}$ & $24 \mathrm{ef}$ & $181.0 \mathrm{~cd}$ \\
\hline 0:200:100 & $262.7 \mathrm{~d}$ & $76.7 \mathrm{~d}$ & $13.0 \mathrm{~d}$ & $26.0 \mathrm{~d}$ & $175.0 \mathrm{e}$ \\
\hline $0: 300: 150$ & $268.3 c$ & $80.7 \mathrm{c}$ & $13.7 \mathrm{~cd}$ & $28.0 \mathrm{c}$ & $167.3 \mathrm{f}$ \\
\hline $5: 0: 0$ & $253.3 \mathrm{f}$ & $68.3 \mathrm{ef}$ & $10.7 \mathrm{ef}$ & 23.0fg & $187.0 \mathrm{~b}$ \\
\hline $5: 100: 50$ & $262.7 \mathrm{~d}$ & $74.7 \mathrm{~d}$ & $13.3 \mathrm{~cd}$ & $25.3 \mathrm{de}$ & $177.3 \mathrm{de}$ \\
\hline $5: 200: 100$ & $269.3 c$ & $81.3 c$ & $14.3 \mathrm{c}$ & $28.0 \mathrm{c}$ & $173.3 \mathrm{e}$ \\
\hline $5: 300: 150$ & $281.3 b$ & $86.3 b$ & $16.0 \mathrm{~b}$ & $30.0 \mathrm{~b}$ & $162.3 \mathrm{~g}$ \\
\hline 10:0:0 & $258.0 \mathrm{e}$ & $70.3 \mathrm{e}$ & $13.7 \mathrm{c}$ & $24.0 \mathrm{ef}$ & $182.3 \mathrm{bc}$ \\
\hline 10:100:50 & $269.7 \mathrm{c}$ & $76.3 d$ & $15.7 \mathrm{~b}$ & $29.0 \mathrm{bc}$ & $173.0 \mathrm{e}$ \\
\hline 10:200:100 & $283.0 \mathrm{~b}$ & $88.0 \mathrm{~b}$ & $17.3 \mathrm{a}$ & $33.3 \mathrm{a}$ & $163.0 \mathrm{fg}$ \\
\hline 10:300:150 & $294.3 \mathrm{a}$ & $92.7 \mathrm{a}$ & $17.7 \mathrm{a}$ & $34.3 \mathrm{a}$ & $120.3 \mathrm{~h}$ \\
\hline \multicolumn{6}{|c|}{$2007 / 2008$} \\
\hline $0: 0: 0$ & $244.3 \mathrm{~h}$ & $66.0 \mathrm{i}$ & $10.7 \mathrm{~g}$ & $22.7 \mathrm{f}$ & $187.3 \mathrm{a}$ \\
\hline 0:100:50 & $253.0 \mathrm{~g}$ & $72.3 \mathrm{gh}$ & $11.7 \mathrm{~g}$ & $24.7 \mathrm{ef}$ & $173.3 \mathrm{c}$ \\
\hline 0:200:100 & $265.0 \mathrm{e}$ & $77.7 \mathrm{ef}$ & $13.0 \mathrm{f}$ & $25.7 \mathrm{de}$ & $163.0 \mathrm{~d}$ \\
\hline $0: 300: 150$ & $269.7 \mathrm{~d}$ & $81.3 \mathrm{~cd}$ & $14.3 \mathrm{e}$ & $28.3 b c$ & $155.7 \mathrm{e}$ \\
\hline $5: 0: 0$ & $253.3 \mathrm{fg}$ & $69.0 \mathrm{hi}$ & $11.3 \mathrm{~g}$ & $24.0 \mathrm{ef}$ & $182.0 \mathrm{~b}$ \\
\hline $5: 100: 50$ & $263.7 e^{\circ}$ & 75.0fg & $14.7 \mathrm{e}$ & $27.3 \mathrm{~cd}$ & $167.0 \mathrm{~d}$ \\
\hline $5: 200: 100$ & $269.7 d$ & $82.7 \mathrm{c}$ & $15.3 \mathrm{de}$ & $28.7 b c$ & $154.3 \mathrm{e}$ \\
\hline $5: 300: 150$ & $280.0 \mathrm{c}$ & $87.7 \mathrm{~b}$ & $16.3 \mathrm{~cd}$ & $29.7 b$ & $149.3 \mathrm{f}$ \\
\hline 10:0:0 & $257.3 \mathrm{f}$ & $71.0 \mathrm{~h}$ & $11.7 \mathrm{~g}$ & $24.7 \mathrm{ef}$ & $171.3 \mathrm{c}$ \\
\hline 10:100:50 & $271.0 \mathrm{~d}$ & $79.0 \mathrm{de}$ & $16.7 \mathrm{bc}$ & $29.7 b$ & $157.3 \mathrm{e}$ \\
\hline 10:200:100 & $284.3 b$ & $87.7 \mathrm{~b}$ & $17.7 \mathrm{ab}$ & $35.0 \mathrm{a}$ & $142.0 \mathrm{~g}$ \\
\hline 10:300:150 & $298.0 \mathrm{a}$ & $93.7 \mathrm{a}$ & $18.7 \mathrm{a}$ & $36.3 \mathrm{a}$ & $118.0 \mathrm{~h}$ \\
\hline
\end{tabular}


in 10:300:150 gm/plant, respectively gave the highest values for those parameters. Moreover, all treatments significantly reduces the No. of days from shooting to harvest in comparison with control in both experimental seasons. Humic materials may also increase root growth in a manner similar to auxins. The previous results agreed with those obtained by Abou-Aziz et al. (1993), Russo et al. (1995), Mayaz (1997), Saad and Attawia (1999), Awaad and El-Shenawi (2005) and Du-Hui Ying et al. (2007).

2. Effect of Humic acid, $\mathrm{K}$ and $\mathrm{Mg}$ fertilization on physical properties of fingers and yield:

As for the effect of Humic acid, $\mathrm{K}$ and $\mathrm{Mg}$ fertilization on yield properties, the data in Table (3) revealed that the rate of 10:300:150 $\mathrm{gm} / \mathrm{plant}$ for $\mathrm{HA}: \mathrm{K}: \mathrm{Mg}$ treatment significantly increase finger diameter, number of fingers per bunch, number of hands per bunch, bunch weight and yield per feddan when compared with all remaining treatments. The data also, showed tendency to increase these parameters gradually with increasing the levels of Humic acid, $\mathrm{K}$ and $\mathrm{Mg}$

\section{Table 3. Effect of Humic acid, $\mathrm{K}$ and $\mathrm{Mg}$ on yield characteristics of GrandNain banana} 2006/2007 and 2007/2008 seasons

\begin{tabular}{lllllll}
\hline $\begin{array}{l}\text { Treatments } \\
\text { (HA:K:Mg) } \\
\text { gm/plant/year }\end{array}$ & $\begin{array}{l}\text { Avg. } \\
\text { diameter } \mathbf{( c m )}\end{array}$ & $\begin{array}{l}\text { No. } \\
\text { finger/bunch }\end{array}$ & $\begin{array}{l}\text { No. of } \\
\text { bunch }\end{array}$ & hands/ & $\begin{array}{l}\text { Bunch } \\
\text { weight (Kg) }\end{array}$ & $\begin{array}{l}\text { Yield/fed. } \\
\text { bunches) (ton) }\end{array}$ \\
\hline & & & $\mathbf{9 0 0 6} / \mathbf{2 0 0 7}$ & & \\
\hline $0: 0: 0$ & $2.60 \mathrm{i}$ & $194.33 \mathrm{i}$ & $9.33 \mathrm{~g}$ & $19.00 \mathrm{i}$ & $17.10 \mathrm{i}$ \\
$0: 100: 50$ & $2.80 \mathrm{hi}$ & $210.00 \mathrm{gh}$ & $10.33 \mathrm{ef}$ & $23.00 \mathrm{~h}$ & $20.70 \mathrm{~h}$ \\
$0: 200: 100$ & $3.03 \mathrm{fg}$ & $216.33 \mathrm{fg}$ & $11.33 \mathrm{~cd}$ & $27.00 \mathrm{ef}$ & $24.30 \mathrm{ef}$ \\
$0: 300: 150$ & $3.30 \mathrm{e}$ & $226.00 \mathrm{de}$ & $11.67 \mathrm{~cd}$ & $30.00 \mathrm{~cd}$ & $27.00 \mathrm{~cd}$ \\
$5: 0: 0$ & $2.87 \mathrm{gh}$ & $20367 \mathrm{~h}$ & $10.00 \mathrm{fg}$ & $22.67 \mathrm{~h}$ & $20.40 \mathrm{~h}$ \\
$5: 100: 50$ & $3.17 \mathrm{ef}$ & $222.67 \mathrm{ef}$ & $11.33 \mathrm{~cd}$ & $24.67 \mathrm{fgh}$ & $22.20 \mathrm{fgh}$ \\
$5: 200: 100$ & $3.67 \mathrm{~d}$ & $230.00 \mathrm{~d}$ & $12.00 \mathrm{c}$ & $28.00 \mathrm{de}$ & $25.20 \mathrm{de}$ \\
$5: 300: 150$ & $3.93 \mathrm{c}$ & $237.67 \mathrm{c}$ & $13.67 \mathrm{~b}$ & $31.67 \mathrm{c}$ & $28.50 \mathrm{c}$ \\
$10: 0: 0$ & $3.00 \mathrm{fgh}$ & $210.33 \mathrm{~g}$ & $11.00 \mathrm{de}$ & $24.00 \mathrm{gh}$ & $21.60 \mathrm{gh}$ \\
$10: 100: 50$ & $3.60 \mathrm{~d}$ & $225.33 \mathrm{de}$ & $13.33 \mathrm{~b}$ & $26.00 \mathrm{efg}$ & $23.40 \mathrm{e}$ \\
$10: 200: 100$ & $4.20 \mathrm{~b}$ & $246.00 \mathrm{~b}$ & $13.67 \mathrm{~b}$ & $35.00 \mathrm{~b}$ & $31.50 \mathrm{~b}$ \\
$10: 300: 150$ & $4.60 \mathrm{a}$ & $266.00 \mathrm{a}$ & $15.00 \mathrm{a}$ & $38.67 \mathrm{a}$ & $34.80 \mathrm{a}$ \\
\hline & & & $\mathbf{2 0 0 7 / 2 0 0 8}$ & & \\
\hline $0: 0: 0$ & $2.67 \mathrm{f}$ & $196.33 \mathrm{~g}$ & $8.33 \mathrm{ef}$ & $20.33 \mathrm{~g}$ & $18.30 \mathrm{~g}$ \\
$0: 100: 50$ & $2.83 \mathrm{f}$ & $211.67 \mathrm{f}$ & $6.67 \mathrm{f}$ & $24.00 \mathrm{ef}$ & $21.60 \mathrm{ef}$ \\
$0: 200: 100$ & $3.17 \mathrm{e}$ & $219.67 \mathrm{e}$ & $11.00 \mathrm{be}$ & $27.33 \mathrm{~cd}$ & $24.60 \mathrm{~cd}$ \\
$0: 300: 150$ & $3.53 \mathrm{~d}$ & $226.00 \mathrm{~d}$ & $11.67 \mathrm{a}-\mathrm{d}$ & $31.33 \mathrm{~b}$ & $28.2 \mathrm{~b}$ \\
$5: 0: 0$ & $2.80 \mathrm{f}$ & $200.67 \mathrm{~g}$ & $9.33 \mathrm{def}$ & $23.00 \mathrm{f}$ & $20.70 \mathrm{f}$ \\
$5: 100: 50$ & $3.23 \mathrm{e}$ & $220.33 \mathrm{e}$ & $11.00 \mathrm{be}$ & $25.67 \mathrm{de}$ & $23.10 \mathrm{de}$ \\
$5: 200: 100$ & $3.63 \mathrm{~d}$ & $232.33 \mathrm{c}$ & $11.67 \mathrm{ad}$ & $29.00 \mathrm{c}$ & $26.10 \mathrm{c}$ \\
$5: 300: 150$ & $3.87 \mathrm{c}$ & $240.00 \mathrm{~b}$ & $13.00 \mathrm{abc}$ & $32.33 \mathrm{~b}$ & $29.10 \mathrm{~b}$ \\
$10: 0: 0$ & $3.13 \mathrm{e}$ & $212.00 \mathrm{f}$ & $10.33 \mathrm{cde}$ & $24.00 \mathrm{ef}$ & $21.60 \mathrm{ef}$ \\
$10: 100: 50$ & $3.63 \mathrm{~d}$ & $228.67 \mathrm{~cd}$ & $13.33 \mathrm{ab}$ & $27.00 \mathrm{~cd}$ & $24.30 \mathrm{~cd}$ \\
$10: 200: 100$ & $4.37 \mathrm{~b}$ & $240.00 \mathrm{~b}$ & $14.00 \mathrm{a}$ & $33.33 \mathrm{~b}$ & $30.00 \mathrm{~b}$ \\
$10: 300: 150$ & $4.70 \mathrm{a}$ & $268.00 \mathrm{a}$ & $14.33 \mathrm{a}$ & $38.33 \mathrm{a}$ & $34.50 \mathrm{a}$ \\
\hline $\mathrm{n}$ & & & &
\end{tabular}

Values with the same letter(s) in each column are not significantly differed at 0.05 levels. 
Table 4. Effect of Humic acid, $\mathrm{K}$ and $\mathrm{Mg}$ on total soluble solids, total acidity, starch and total sugars percentages of GrandNain banana 2006/2007 and 2007/2008 seasons

\begin{tabular}{|c|c|c|c|c|}
\hline $\begin{array}{l}\text { Treatments } \\
\text { (HA:K:Mg) } \\
\text { gm/plant/year }\end{array}$ & T.S.S. \% & Total acidity \% & Starch \% & Total sugars \% \\
\hline \multicolumn{5}{|c|}{$2006 / 2007$} \\
\hline 0:0:0 & $14.80 \mathrm{~g}$ & $0.343 \mathrm{a}$ & $1.35 \mathrm{f}$ & $14.49 \mathrm{i}$ \\
\hline 0:100:50 & $15.23 \mathrm{fg}$ & $0.297 \mathrm{~b}$ & $1.41 \mathrm{de}$ & $15.18 \mathrm{~h}$ \\
\hline 0:200:100 & $16.03 \mathrm{e}^{\mathrm{e}}$ & $0.277 \mathrm{~d}$ & $1.55 \mathrm{c}$ & $16.18 \mathrm{ef}$ \\
\hline $0: 300: 150$ & $16.47 \mathrm{de}$ & $0.237 \mathrm{~h}$ & $1.58 \mathrm{c}$ & $16.88 \mathrm{~d}$ \\
\hline $5: 0: 0$ & $15.33 \mathrm{f}$ & $0.283 \mathrm{c}$ & $1.36 \mathrm{ef}$ & 14.98hi \\
\hline 5:100:50 & $16.13 \mathrm{e}$ & $0.270 \mathrm{e}$ & $1.46 \mathrm{~d}$ & $15.68 \mathrm{fg}$ \\
\hline $5: 200: 100$ & $16.83 \mathrm{~cd}$ & $0.243 \mathrm{~g}$ & $1.60 \mathrm{c}$ & $16.38 \mathrm{de}$ \\
\hline $5: 300: 150$ & $17.73 b$ & $0.223 \mathrm{j}$ & $1.68 \mathrm{~b}$ & $17.54 \mathrm{c}$ \\
\hline 10:0:0 & $16.13 \mathrm{e}$ & $0.257 \mathrm{f}$ & $1.40 \mathrm{ef}$ & $16.63 \mathrm{de}$ \\
\hline 10:100:50 & $17.20 \mathrm{c}$ & $0.230 \mathrm{i}$ & $1.56 \mathrm{c}$ & $17.54 \mathrm{c}$ \\
\hline 10:200:100 & $18.03 b$ & $0.207 \mathrm{k}$ & $1.72 b$ & $19.27 \mathrm{~b}$ \\
\hline $10: 300: 150$ & $20.50 \mathrm{a}$ & 0.1831 & $1.81 \mathrm{a}$ & $21.67 \mathrm{a}$ \\
\hline \multicolumn{5}{|c|}{$2007 / 2008$} \\
\hline $0: 0: 0$ & $15.40 \mathrm{~h}$ & $0.393 \mathrm{a}$ & $1.34 \mathrm{~h}$ & $15.27 \mathrm{~h}$ \\
\hline 0:100:50 & $15.67 \mathrm{gh}$ & $0.370 \mathrm{~b}$ & $1.42 \mathrm{fg}$ & $15.53 \mathrm{gh}$ \\
\hline $0: 200: 100$ & $16.37 f$ & $0.350 \mathrm{~d}$ & $1.52 \mathrm{e}^{\circ}$ & $16.43 \mathrm{def}$ \\
\hline $0: 300: 150$ & $16.80 \mathrm{e}$ & $0.290 \mathrm{~g}$ & $1.64 \mathrm{~cd}$ & $16.87 d$ \\
\hline $5: 0: 0$ & $15.80 \mathrm{~g}$ & $0.370 \mathrm{~b}$ & $1.39 \mathrm{gh}$ & $15.97 \mathrm{fg}$ \\
\hline $5: 100: 50$ & $16.47 \mathrm{ef}$ & $0.350 \mathrm{c}$ & $1.46 \mathrm{f}$ & $16.21 \mathrm{ef}$ \\
\hline $5: 200: 100$ & $17.63 d$ & $0.310 \mathrm{e}$ & $1.62 \mathrm{~cd}$ & $16.74 \mathrm{de}$ \\
\hline $5: 300: 150$ & $18.70 \mathrm{c}$ & $0.260 \mathrm{~h}$ & $1.67 \mathrm{c}$ & $17.87 \mathrm{c}$ \\
\hline 10:0:0 & $16.77 \mathrm{e}$ & $0.310 \mathrm{f}$ & $1.41 \mathrm{fg}$ & $16.97 \mathrm{~d}$ \\
\hline 10:100:50 & $19.70 \mathrm{~d}$ & $0.240 \mathrm{i}$ & $1.62 \mathrm{~d}$ & $18.33 \mathrm{c}$ \\
\hline $10: 200: 100$ & $20.00 \mathrm{~b}$ & $0.220 \mathrm{j}$ & $1.75 \mathrm{~b}$ & $20.53 b$ \\
\hline 10:300:150 & $22.53 \mathrm{a}$ & $0.160 \mathrm{k}$ & $1.83 \mathrm{a}$ & $22.33 a$ \\
\hline
\end{tabular}

Values with the same letter(s) in each column are not significantly differed at 0.05 levels.

were positively affected by the combined application of humic acid, potassium sulphate and magnesium sulphate compared with control treatment. Fruit quality was greatly improved in terms of increasing total soluble solids, starch, as well as total sugars and decreasing acidity due to the application of the three materials. The promotion on fruit quality was associated with the increase in concentrations of each compound. Fruit quality was improved by increasing humic acid, potassium sulphate and magnesium sulphate concentrations, the best treatment was (10, 300 and 150 gm/plant), respectively. Untreated plants (control) showed the lowest fruit quality in both experimental seasons and the differences were statistically significant with this treatment and the other remaining treatments. These results, completely, agreed with those obtained by Li (1999), Saad and Atawia (1999), Itoo and Manivannan (2004) and Abd El-Mawgoud et al. (2007).

4. Effect of Humic acid, $\mathrm{K}$ and Mg fertilization on $\mathrm{N}$, $\mathrm{P}, \mathrm{K}$ and $\mathrm{Mg}$ percentage:
Table (5) showed that increasing $\mathrm{HA}, \mathrm{K}$ and $\mathrm{Mg}$ levels was followed by gradual significant increase in the percentages of $\mathrm{K}$ and $\mathrm{Mg}$ in the leaves. The highest values were recorded for treatment $\mathrm{HA}: \mathrm{K}: \mathrm{Mg}$ at 10:300:150 gm/plant. The minimum values were detected on the untreated plants. The concentration of nitrogen in banana leaves was not affected by humic acid, potassium sulphate and magnesium sulphate during both experimental seasons. This may be due to better growth and yield attained by $\mathrm{Mg}$ rates and number of application. The different fertilization treatments caused significant changes in the leaf phosphorus content and the highest values were found in humic acid $5 \mathrm{gm}$ with potassium sulphate 0,100 and 200 and magnesium sulphate 0,50 and $100 \mathrm{gm} /$ plant in the first season, whereas in the second season the highest values were recorded for treatments $\mathrm{HA}: \mathrm{K}: \mathrm{Mg}$ in rates 0:100:50 and 0:200:100 gm/plant. Similar observation was obtained by Abd El-Kader et al. (1994), Mayaz (1997), Abd ElNaby (2000), Fallahi et al. (2006), Farag (2006) and DuHui Ying et al. (2007). 
Table 5. Effect of Humic acid, $\mathrm{K}$ and $\mathrm{Mg}$ on $\mathrm{N}, \mathrm{P}, \mathrm{K}$ and $\mathrm{Mg}$ percentages in the leaves of GrandNain banana 2006/2007 and 2007/2008 seasons

\begin{tabular}{|c|c|c|c|c|}
\hline $\begin{array}{l}\text { Treatments(HA:K:Mg) } \\
\text { gm/plant/year }\end{array}$ & $\mathbf{N} \%$ & P \% & K \% & Mg \% \\
\hline \multicolumn{5}{|c|}{$2006 / 2007$} \\
\hline $0: 0: 0$ & $3.23 \mathrm{a}$ & $0.217 \mathrm{~d}$ & $3.40 \mathrm{~g}$ & 0.2931 \\
\hline 0:100:50 & $3.20 \mathrm{a}$ & $0.217 \mathrm{~d}$ & $3.80 \mathrm{ef}$ & $0.363 \mathrm{i}$ \\
\hline 0:200:100 & $3.17 \mathrm{a}$ & $0.233 b$ & $4.00 \mathrm{de}$ & $0.377 \mathrm{~g}$ \\
\hline 0:300:150 & $3.17 \mathrm{a}$ & $0.220 c$ & $4.37 \mathrm{c}$ & $0.397 \mathrm{e}$ \\
\hline $5: 0: 0$ & $3.17 \mathrm{a}$ & $0.227 \mathrm{a}$ & $3.63 \mathrm{fg}$ & $0.307 \mathrm{k}$ \\
\hline $5: 100: 50$ & $3.20 \mathrm{a}$ & $0.227 \mathrm{a}$ & $4.13 \mathrm{~cd}$ & $0.373 \mathrm{~h}$ \\
\hline $5: 200: 100$ & $3.13 \mathrm{a}$ & $0.227 \mathrm{a}$ & $4.23 \mathrm{~cd}$ & $0.393 \mathrm{f}$ \\
\hline $5: 300: 150$ & $3.20 \mathrm{a}$ & $0.223 b$ & $4.80 \mathrm{~b}$ & $0.420 \mathrm{c}$ \\
\hline 10:0:0 & $3.17 \mathrm{a}$ & $0.213 \mathrm{e}$ & $3.80 \mathrm{ef}$ & $0.337 \mathrm{j}$ \\
\hline 10:100:50 & $3.17 \mathrm{a}$ & $0.210 \mathrm{f}$ & $4.10 \mathrm{~d}$ & $0.403 d$ \\
\hline 10:200:100 & $3.13 \mathrm{a}$ & $0.217 \mathrm{~d}$ & $4.70 \mathrm{~b}$ & $0.437 \mathrm{~b}$ \\
\hline 10:300:150 & $3.17 \mathrm{a}$ & $0.220 \mathrm{c}$ & $5.10 \mathrm{a}$ & $0.460 \mathrm{a}$ \\
\hline \multicolumn{5}{|c|}{$2007 / 2008$} \\
\hline $0: 0: 0$ & $3.40 \mathrm{a}$ & $0.220 \mathrm{c}$ & $3.30 \mathrm{~h}$ & 0.3171 \\
\hline 0:100:50 & $3.37 \mathrm{a}$ & $0.227 \mathrm{a}$ & $3.67 \mathrm{fg}$ & $0.357 \mathrm{j}$ \\
\hline $0: 200: 100$ & $3.37 \mathrm{a}$ & $0.227 \mathrm{a}$ & $3.90 \mathrm{de}$ & $0.390 \mathrm{~h}$ \\
\hline 0:300:150 & $3.33 \mathrm{a}$ & $0.223 b$ & $4.13 c$ & $0.413 \mathrm{f}$ \\
\hline $5: 0: 0$ & $3.37 \mathrm{a}$ & $0.223 b$ & $3.57 \mathrm{~g}$ & $0.343 \mathrm{k}$ \\
\hline $5: 100: 50$ & $3.33 \mathrm{a}$ & $0.223 b$ & $3.83 \mathrm{ef}$ & $0.397 \mathrm{~g}$ \\
\hline $5: 200: 100$ & $3.40 \mathrm{a}$ & $0.220 c$ & $4.07 \mathrm{~cd}$ & $0.440 \mathrm{~d}$ \\
\hline $5: 300: 150$ & $3.30 \mathrm{a}$ & $0.220 c$ & $4.60 \mathrm{~b}$ & $0.453 \mathrm{c}$ \\
\hline 10:0:0 & $3.40 \mathrm{a}$ & $0.223 b$ & 4.77ef & $0.370 \mathrm{i}$ \\
\hline 10:100:50 & $3.33 \mathrm{a}$ & $0.220 \mathrm{c}$ & $4.13 c$ & $0.433 \mathrm{e}$ \\
\hline $10: 200: 100$ & $3.67 \mathrm{a}$ & $0.220 \mathrm{c}$ & $4.63 b$ & $0.457 \mathrm{~b}$ \\
\hline 10:300:150 & $3.67 \mathrm{a}$ & $0.220 \mathrm{c}$ & $4.83 \mathrm{a}$ & $0.500 \mathrm{a}$ \\
\hline
\end{tabular}

Values with the same letter(s) in each column are not significantly differed at 0.05 levels.

\section{CONCLUSION}

Generally, it could be concluded that the treatment of $(10 \mathrm{gm} \quad \mathrm{HA}: \mathrm{K}: \mathrm{Mg}$ at 10:300:150 gm/plant, respectively, seems to be the promising treatment to produce the highest growth, physical properties of fingers and yield, finger chemical properties and leaf mineral content of GrandNain banana under the above experimental conditions. It will keep out environment pollution and fertilizers lost from applying high fertilizer rates.

\section{REFERENCES}

Abd El-Kader, A.M.M.; F.M.B. El-Makhoun and A.A. Abd El-Al (1994). Effect of $\mathrm{N}$ and $\mathrm{K}$ fertilization on growth and yield of GrandNain banana cultivar. Zagazig J. Agric. Res. 21(5) 1489-1495.

Abd El-Naby, S.K.M. (2000). Effect of banana compost as organic manure on growth, nutrients status, yield and fruit quality of Maghrabi banana. Assiut J. of Agric. Sci. 3:101-114.

Abd El-Naby, S.K.M. and A.M.Gomma (2000). Growth, nutritional status, yield and fruit quality of Maghrabi banana as affected by some organic manures and biofertilizer. Minufiya J. Agric. Res., 25(4): 1113-1129.

Abd El-Mawgoud, A.M.R.; N.H.M. El-Greadly; Y.I. Helmy and S.M. Singer (2007). Responses of tomato plants to different rates of humic-based fertilization. Journal of Applied Sciences Research 2007; Feb.:169-174.

Abou-Aziz, A.B.; M.M. Abd El-Kader; M.R. El-Sonbaty and M.M. Saad (1993). Effect of different levels (K-MAG) compound fertilizer on vegetative growth, yield, fruit quality and some leaf nutrient contents of "Maghrabi" banana cultivar. J. Agric. Sci. Assiut. Vol. 24: 1, 45-57.

Ahmed, F.F. and M.H. Morsy (1999). A new method for measuring leaf area in different fruit species. Minia J. Agric. Res. \& Develop. Vol. (19) pp 97-105.

A.O.A.C. (1995). Official Methods of Analysis, published by A.O.A.C., Box 540 and Washington, D.C.

Awad A.M. and M.R. El-Shenawi (2005). Integrated production technique for banana "Williams" using organic and mineral NK fertilization in calcareous soils. Assiut Journal of Agric.Sci., Vol. 36, No. 4, 21-43.

Donnell, R.W. (1973). The auxin-like effects of humic preparations from leonardite. Soil Sci., 116:106-112. 
Du-Hui Ying; Xue-Shi Chuan and Sun-Zhong Fu (2007). Effects of different application rates of humic acid compound fertilizer on leaf nutrient accumulation and physiological mechanism of grape. Zhonggu Shengtai Nongye Xuebao. Chinese Journal of Eco Agriculture. 15(1): 49-51.

El-Shenawi, M.R. (2000). Effect of nitrogen and potassium fertilization on growth, yield, fruit quality and leaf mineral content of Williams banana grown in calcareous soil. Ph.D. Thesis. Fac. Of Agric. Alex. Univ.

El-Shenamwi, M.R. and El-Sayed, S.A.M. (2005). Effect of bio and organic fertilization on growth, productivity, fruit quality and leaf mineral content of GrandNain banana. J. Adv. Agric. Res. (Fac. Agric. Saba Basha). Vol. 10(3): 779-789.

El-Shenawi, I.E. and T.A. Fayed (2005). Evaluation of the conventional to organic and biofertilizers on "Crimson seedless" Grapevines in comparison with chemical fertilizers. II. Yield and fruit quality. Egypt. J. App. Sci., 20(1): 212-225.

Fallahi, E; B. Fallahi and M.M Seyedbagheri (2006). Influence of humic substances and nitrogen on yield, fruit quality and leaf mineral elements of early Spur Rome' apple. Journal of Plant Nutrition. 2006; 29(10): 18191833.

FAO. (1980). Soil and plant analysis as basis of fertilizer recommendations. Cottenie, A. (Ed.); Soils bulletin No. 38(2). Rome.

Farag, S.G. (2006). Minimizing mineral fertilizers in grapevine farms to reduce the chemical residuals in grapes. M.Sc. Thesis, Institute of Environmental Studies \& Research, Ain Shams University, Egypt, pp: 67.

Gomaa, A.M. and S.K.M. Abd El-Naby. (2000). Biofertilization of Maghrabi banana corms and its influence on Mycorrhizal flungi infection and vegetative growth parameters. Minufiya J. Agric. Res., 25(4): 11311144.

Hewit, G.W. (1955). Leaf analysis as guide to the nutrition of bananas. Emp. J. Exp. Agric. Vol. 23 (Hort. Abst., 25: 4346).

Itoo, B.A. and K. Manivannan (2004). Effect of macro and micronutients in different forms in comparison with humic acid on growth, yield and quality of tomato (Lycopersicon esculentum Mill.) cv. Annapurna. South Indian Horticulture. 2004; 52(1/6): 342-346.

Lahave, E. (1976). The manure requirements of the "Ziv" (Williams Hybride) banana cultivar Division of subtropical Horticulture, special publication, Agricultural Research Organization, No. 65, 115-118.Israel.

Lahave, E; M. Bareket and D. Zamit (1981). The value of $\mathrm{KNO}_{3}$, organic manure and poly-feed in banana plantation under drip irrigation. Fruits. 36(3) 151-155.

Lahave, E. and D.W. Turner. (1989). Banana nutrition, fertilizing for high yield. IPI bulletin No. 7. International Potash Institute. Berne/Switzerlan.
Li, N.; X.X. Wang and B.L. Lu (1999). Study of the effect of apple liquid fertilizer on the growth and fruit development of Starkrimson apple variety. (in Chinese). China Fruits No. 4: 20-21 (c.f. Hort. Abst. 70, 5: 3628).

Liu, C.; R.J. Cooper and D.C. Bowman (1998). Humic acid application effects photosynthesis, root development and nutrient content of creeping bent grass. HortScience, 33 (6) $1023-1025$.

Mayaz, M. (1997). Effect of organic and inorganic forms of potassium fertilization on productivity of Dwarf Cavendish banana plants (Hindi cv.) in sandy soil. Egyptian J. of Applied Science, 2(6): 187-205.

Nijjar, G.S. (1985). Nutrition of Fruit Trees. Mrs Usha Raj Kumar for Kalyani Publishers New Delhi India, 306-308.

Purchase J. (1997). Humus, humic acid and natural chelating agents. jpp/inforamp.net.

Russo, R.O. and G.P. Berlyn (1990). The use of organic biostimulants to help low input sustainable agriculture. J. Sustainable Agric., 1(2): 19-42.

Russo, R; J. Lugo; O. Arreola and O. Arango (1995). Effect of a humic bio-stimulant extracted from the banana rachis on the growth of banana seedling (Musa AAA "Cavendish" subgroup "Cran enano" clone). Agronomia Mesoamericana.; 6: 130-133.

Saad M.M. and A.A.R. Atawia (1999). Effect of potash application on growth, yield and fruit quality of GrandNain banana in sandy soil under drip irrigation system. Alex. J. Agric. Res., 44(1): 171-180.

Saleh, M.M.S. (1996). Effect of fertilization with different forms of nitrogen fertilizers on growth, flowering mineral content and yield of banana. Ph. D. Thesis Fac. Of Agric., Ain Shams Univ. Cairo, Egypt.

Saleh, M.M.S.; S. El-Ashry and A.M. Gomaa (2006). Performance of tompson seedless grapevine as influenced by organic fertilizer humic acid and biofertilizers under sandy soil conditions. Research J. of Agric. And Biological Sciences, 2(6): 467-471.

Senn, T.L. and A.R. Kingman (1973). A review of humus and humic acids. South Carolina Agricultural Experiment Station, Clemson, SC. Research Series Report No. 145.

Snedecor, C.W. and W.G. Cochran (1990). Statistical methods $7^{\text {th }}$ ed. The Iowa State Univ. Press. Ames Iowa. USA. P. 593.

Steel, R.G.D. and G.H. Torrie. 1982. Principles and procedures of Statistics. A biometrical Approach. McGraw-Hill Book Co., 625.

Tatini, M.; P. Bertoni; A. Landi and M.L. Traversi (1991). Effect of humic acids on growth and biomass portioning of container grown olive plants. Acta. Hort. 294: 75:80.

Westerman, R.L. (ed.) (1990). Soil testing and plant analyisis. Third edition, Soil Sci. Soc. Am., Madison, Wisconsin US.A.

Zhang, L.X., and Aro, E.-M. (2002). Synthesis, membrane insertion and assembly of the chloroplast-encoded D1 protein into photosystem II. FEBS Lett. 512, 13-18. 


\section{الملخص العربي}

\section{أستجابة نباتات الموز صنف "جراندنان" بالتسميد بحمض الميوميك والبوتاسيوم والمفنسيوم}

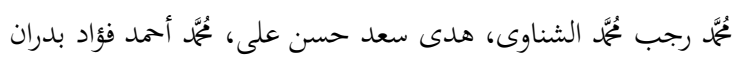

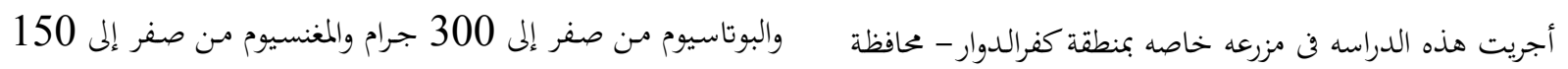

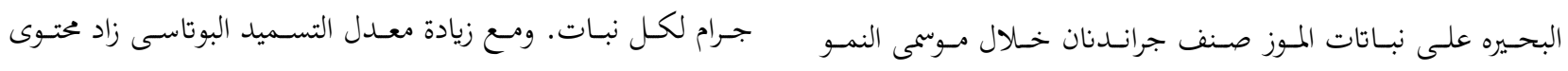

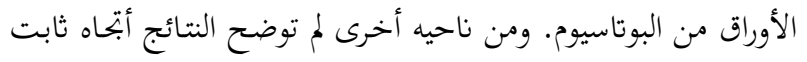

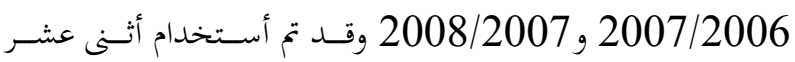

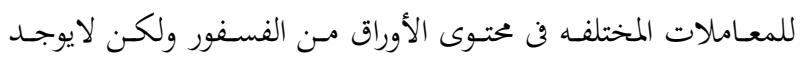

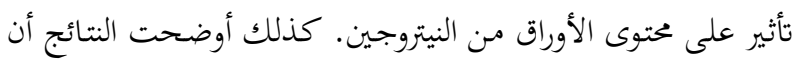

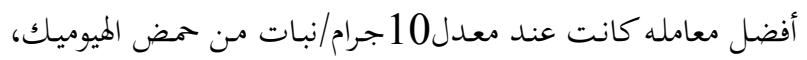
300جرام من كبريتات البوتاسيوم، 150 جرام من كبريتات المغنسيوم على التوالى في جميع الصفات التى تم دراستها. معامله من حمض الهيوميك البوتاسيوم:المغنسيوم.

أوضحت نتـائج الدراسه أن هنـاك أختلافات كبيره في صفات

النمووالمحصول والخصائص الطبيعيه والكيماويه للثمار والمختوى المعددنى للأوراق بإختلاف نسبة الهيوميك والبوتاسيوم والمغنسيوم وكانت هناك زيادة تدريجيـه واضـحه في كـل الصـفات تحست الدراسـة عنســ زيادة

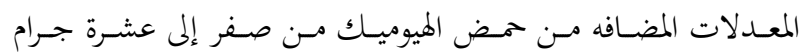

